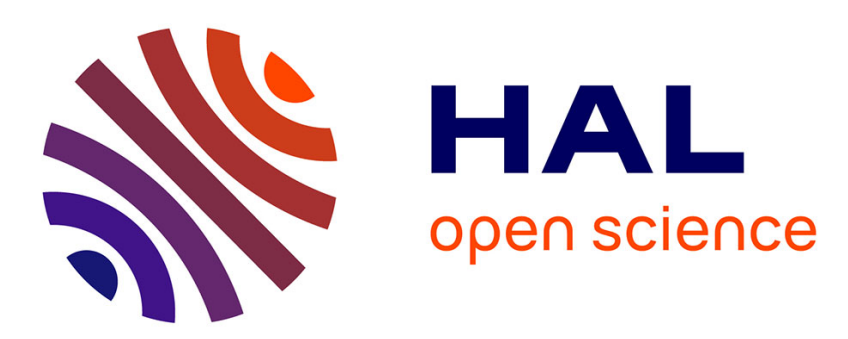

\title{
A critical look into Rayleigh damping forces for seismic performance assessment of inelastic structures
}

\author{
Pierre Jehel
}

\section{To cite this version:}

Pierre Jehel. A critical look into Rayleigh damping forces for seismic performance assessment of inelastic structures. Engineering Structures, 2014, 78, pp.28-40. 10.1016/j.engstruct.2014.08.003 . hal-01053896

\section{HAL Id: hal-01053896 https://hal.science/hal-01053896}

Submitted on 4 Aug 2014

HAL is a multi-disciplinary open access archive for the deposit and dissemination of scientific research documents, whether they are published or not. The documents may come from teaching and research institutions in France or abroad, or from public or private research centers.
L'archive ouverte pluridisciplinaire HAL, est destinée au dépôt et à la diffusion de documents scientifiques de niveau recherche, publiés ou non, émanant des établissements d'enseignement et de recherche français ou étrangers, des laboratoires publics ou privés. 


\title{
A CRITICAL LOOK INTO RAYLEIGH DAMPING FORCES FOR SEISMIC PERFORMANCE ASSESSMENT OF INELASTIC STRUCTURES
}

\author{
Pierre Jehel ${ }^{12}$ \\ pierre.jehel[at]ecp.fr
}

August 2 ${ }^{\text {nd }}, 2014$ : Paper accepted for publication in Engineering Structures

\begin{abstract}
Rayleigh damping forces are commonly introduced in the numerical simulations of nonlinear structures run to assess structural performance in case of an earthquake. Their purpose is to account for energy dissipative mechanisms not otherwise explicitly represented in the model. When caused by interactions between the structure and its surrounding environment, energy dissipation is external to the structure, whereas it is internal when resulting from energy absorption mechanisms activated in the structure. In this paper, the concept of discrepancy forces is introduced in the framework of computational dynamics. Then, damping forces are presented as a model of these so-called discrepancy forces to represent internal energy dissipation. On the other hand, the discrepancy forces are identified from a set of experimental data recorded during shaking-table test of a ductile moment-resisting frame, which provides the rationale for a critical look into Rayleigh damping forces. It is in particular observed that, for the structure tested, the Rayleigh damping model used is inaccurate as a representation of the discrepancy forces. Besides, while the knowledge of the discrepancy forces allows for rationally discussing the capabilities of the inelastic structural model to represent the actual behavior of the structure, this is only possible to a limited extent with the Rayleigh damping model used.
\end{abstract}

keywords: seismic performance, damping forces, nonlinear structure, computational mechanics, experimental test design, earthquake engineering.

\section{INTRODUCTION}

1.1. Seismic structural performance assessment. Seismic performance assessment of inelastic structures is a key step in a seismic risk management process that aims at mitigating the risks for the populations and the infrastructures in seismically active regions. Seismic risk is a combination of seismic hazard and

\footnotetext{
${ }^{1}$ Laboratoire MSSMat / CNRS-UMR 8579, École Centrale Paris, Grande voie des Vignes, 92295 Châtenay-Malabry Cedex, France

${ }^{2}$ Department of Civil Engineering and Engineering Mechanics, Columbia University, $630 \mathrm{SW}$ Mudd, 500 West 120th Street, New York, NY, 10027, USA
} 
structural vulnerability and can be effectively formalized and communicated in a probabilistic setting (see e.g. [6]):

$$
P_{\mathrm{PL}}=\int P\left[\mathrm{EDP} \geq \mathrm{EDP}_{\mathrm{PL}} \mid \mathrm{IM}=x\right] \cdot P[\mathrm{IM}=x] d x
$$

In this equation, PL is the performance level associated to a certain value of an engineering demand parameter (EDP) of interest. For instance, in the case of moment-resisting frame structures, maximum interstory drift is often used as the EDP of interest (see $[6,16,5]$ among others) because it is possible to map it to meaningful PL such as "immediate occupancy", "structural damage" or "collapse prevention" [6]. In equation (1), IM refers to the intensity measure of the seismic ground motion, and the probability to observe an earthquake with an IM equal to $x$ in the region of interest, that is $P[\operatorname{IM}=x]$, is given by seismic hazard maps. This paper focusses on the role of Rayleigh damping forces in the vulnerability assessment of nonlinear structures, that is on the computation of the conditional probability that a structural performance criteria is exceeded given a certain IM of the ground motion, when the EDPs are computed through time-history analyses.

1.2. Uncertainties in the performance assessment. Whether they are pertaining to the ground motion signal or to the structural response, uncertainties are numerous and can dramatically impact the conclusions of seismic risk analyses. To identify which of the potential uncertainty sources have to be accounted for in the communication of risk analyses results, a series of sensitivity analyses has been conducted in the structural earthquake engineering community (e.g. [16, 5, 18, 15, 24, 2, 23] among others). In particular, the studies in $[18,15,2]$ explicitly account for Rayleigh damping as a potential contributor to the uncertainty in the EDP of interest.

The sources of uncertainty that most strongly affect the repair cost in an earthquake have been sought in [18] using sensitivity analyses. A high-rise reinforced concrete nonductile moment-resisting frame is studied. Structural Rayleigh damping ratio is found to be a minor source of uncertainty in the adopted structural performance measure with respect to the capacity of the structural elements to damage and the seismic ground motion intensity.

In [15], the authors use FOSM method to investigate the sensitivity of a series of EDPs to uncertain parameters among which Rayleigh damping ratio. The building studied is a seven-story reinforced concrete shear-wall structure. It is concluded that, for the local EDPs considered (curvature in critical sections), viscous damping is the second most significant source of uncertainty after the intensity of the ground motion.

In [2], a sensitivity analysis of the maximum interstory drift to inelastic frame element properties, beam-column joint properties as well as structural viscous damping ratio is performed for a reinforced concrete frame structure at various seismic hazard levels. Although the uncertainty in the ground motion dominates 
the overall uncertainty in the interstory drift, Rayleigh damping ratio is found to be one of the most significant other contributors to the EDP of interest.

1.3. Objective and scope of the paper. On the one hand, it has been observed that Rayleigh damping can be a significant contributor to the overall uncertainty in the EDPs of interest for seismic performance assessment of inelastic structures $[15,2]$. On the other hand, it has been shown that using Rayleigh damping forces along with an inelastic structural model can be problematic and lead to unintended consequences that can compromise the validity of the analyses outputs $[10,3]$. Therefore, the objective of this paper is to provide a rational discussion on the validity of Rayleigh damping forces in the time history analyses of inelastic structures and to shed light on a potential strategy to model realistic damping forces in inelastic simulations along with improving the predictive capabilities of the structural models.

Rayleigh damping can be used in seismic simulations either to account for energy dissipation mechanisms that are external to the structure or for energy absorption mechanisms that are internal to the structure. This work focusses on internal energy absorption only. Besides, in case internal energy absorption has to be modeled, we adopt the viewpoint of Rayleigh damping forces being added to complete the seismic energy absorption capacity of the inelastic structural model. In other words, Rayleigh damping forces are not considered in this paper as intrinsic to the structural response but as some ad hoc correction of deficiencies of the inelastic structural model to accurately represent the actual structural response to the seismic action.

The approach adopted in this work is fundamentally different from what is developed in studies focused on structural system identification (see e.g. [9, 20, 21]). In these latter studies, the structure is considered as a system that modifies the seismic ground motion (input signal) into the data (e.g. displacements) recorded at monitored points (output signals). In such analyses, there is no explicit inelastic structural model used to simulate the structural response: the structural system is represented by linear differential equations characterized by modal damping ratios and frequencies that can be identified in the process. Hereafter however, an inelastic structural model is constructed to approximate the response of the structure, and the damping forces time history is identified.

1.4. Outline of the paper. The outline of the paper is as follows. In the next section, basic equations of nonlinear dynamics are first recalled as a baseline for introducing the concept of discrepancy forces in the framework of computational nonlinear dynamics. In particular, the need for experimental data to calculate these discrepancy forces is pointed out. Section 3 is devoted to a short description of the shaking table tests during which the experimental data that are used thereafter were recorded. Then, two inelastic structural models of the tested momentresisting reinforced concrete frame are presented. They are developed using fiber 
frame elements and simple inelastic beam-to-column connections. The discrepancy forces are calculated for both structural models in sections 5 and 6 . How discrepancy forces can be used to improve structural models is in particular discussed and used to parameterize the improved structural model used in section 6 . Before closing the paper with some conclusions, section 7 presents a critical discussion on Rayleigh damping forces based on the rationale provided by the knowledge of the discrepancy forces.

\section{DAMPing FORCES REVISITED - DiscRePANCY FORCES}

2.1. Classical computational nonlinear dynamics. We assume that the dynamic nonlinear structural problem is cast in a standard finite element form. We also assume that displacement (hysteresis), velocity (viscosity), and accelerationproportional (inertia) forces contribute to the structural response (left-hand side of the equation):

$$
\mathbf{M} \ddot{\mathbf{u}}\left(t_{n}\right)+\mathbf{C}\left(t_{n}\right) \dot{\mathbf{u}}\left(t_{n}\right)+\mathbf{F}^{\text {hys }}\left(\mathbf{u} ; t_{n}\right)=\mathbf{F}^{\text {ext }}\left(t_{n}\right)
$$

where $\mathbf{M}$ and $\mathbf{C}$ are the mass and damping matrices, $\mathbf{F}^{\text {hys }}$ is the structural hysteretic restoring force vector, and $\mathbf{F}^{e x t}$ is the external loading vector. $t_{n} \in \mathcal{T}$ with $\mathcal{T}=\{n \times \Delta t \mid n \in[0,1, . ., N], \Delta t=T / N>0\}$ is a discrete process. $\mathbf{F}^{\text {ext }}$ typically consists of the static loadings (dead and service loads), the forces induced by the seismic ground motion, and the reactions at the connections between the structure and its environment. Also, if some energy dissipation sources that are external to the structure are present in the system (structure equipped with energy dissipation device that has known physical properties), they are considered here to act as external loading, so that the viscous and hysteretic forces only account for mechanisms that are internal to the structure.

Because the structural response is possibly nonlinear, we rewrite equation (2) as a residual vector $\mathbf{R}$ that has to be iteratively set to zero:

$$
\mathbf{R}\left(\mathbf{u}, \dot{\mathbf{u}}, \ddot{\mathbf{u}} ; t_{n}\right)=\mathbf{0}
$$

At iteration $k$, with the subscript $n$ referring to $t_{n}$, the Newton-Raphson updating residual reads

$$
\mathbf{R}_{n}^{(k+1)}=\mathbf{R}_{n}^{(k)}+\left.\frac{\mathrm{d} \mathbf{R}}{\mathrm{d} \mathbf{u}}\right|_{n} ^{(k)} \mathrm{d} \mathbf{u}_{n}^{(k)}=\mathbf{0}
$$

where:

$$
\mathbf{R}_{n}^{(k)}=\mathbf{F}_{n}^{e x t}-\mathbf{M} \ddot{\mathbf{u}}_{n}^{(k)}-\mathbf{C}_{n} \dot{\mathbf{u}}_{n}^{(k)}-\mathbf{F}^{h y s}\left(\mathbf{u}_{n}^{(k)}\right)
$$

and the total tangent matrix

$$
\begin{aligned}
\mathbf{S}_{n}^{(k)}=-\left.\frac{\mathrm{d} \mathbf{R}}{\mathrm{d} \mathbf{u}}\right|_{n} ^{(k)} & =-\left.\frac{\partial \mathbf{R}}{\partial \mathbf{u}}\right|_{n} ^{(k)}-\left.\frac{\partial \mathbf{R}}{\partial \dot{\mathbf{u}}}\right|_{n} ^{(k)} \frac{\mathrm{d} \dot{\mathbf{u}}}{\mathrm{d} \mathbf{u}}-\left.\frac{\partial \mathbf{R}}{\partial \ddot{\mathbf{u}}}\right|_{n} ^{(k)} \frac{\mathrm{d} \ddot{\mathbf{u}}}{\mathrm{d} \mathbf{u}} \\
& =\mathbf{K}_{n}^{(k)}+c_{C} \mathbf{C}_{n}^{(k)}+c_{M} \mathbf{M}
\end{aligned}
$$


where $\mathbf{K}=\mathrm{d} \mathbf{F}^{\text {hys }} / \mathrm{d} \mathbf{u}$ is the structural tangent stiffness matrix, $c_{C}=\mathrm{d} \dot{\mathbf{u}} / \mathrm{d} \mathbf{u}$ and $c_{M}=\mathrm{d} \ddot{\mathbf{u}} / \mathrm{d} \mathbf{u}$ are coefficients dependent on both the time step $\Delta t$ and the parameters of any one-step time integration algorithm.

2.2. Damping forces revisited. The presence of velocity-proportional forces in equation (2) does not result from continuum mechanics principles but from the introduction of viscous forces at a structural level. These latter forces are referred to as damping forces because they are designed for representing damping features resulting from some viscous mechanisms activated during the dynamic response of the structure. In earthquake engineering, there is few evidence that actual structural damping results from viscous phenomena and several researchers have claimed that the energy dissipative phenomena that contribute to the overall structural damping should rather be explicitly accounted for in the hysteretic response $\mathbf{F}^{\text {hys }}(t)$ (e.g. [3]).

A shift of perspective is proposed here: Instead of adding viscous forces in the equations of equilibrium, discrepancy forces $\mathbf{F}^{\text {dis }}(t)$ are added. Discrepancy forces not necessarily are proportional to the velocity. They have to be understood as completing the hysteresis and inertia forces in the balance equation so that the model accurately predicts the displacements and accelerations that would be experimentally observed. Then, denoting $\mathbf{y}$ and $\ddot{\mathbf{y}}$ the displacements and accelerations that would actually be observed during experimental tests, the residual vector and total tangent stiffness matrix, compare with equations (5) and (6), now read:

$$
\begin{aligned}
\mathbf{0}:= & \tilde{\mathbf{R}}_{n}=\mathbf{F}_{n}^{e x t}-\mathbf{M} \ddot{\mathbf{y}}_{n}-\mathbf{F}_{n}^{\text {dis }}-\mathbf{F}^{\text {hys }}\left(\mathbf{y}_{n}\right) \\
& \tilde{\mathbf{S}}_{n}=\mathbf{K}_{n}+c_{M} \mathbf{M}
\end{aligned}
$$

2.3. Discrepancy forces. The comparison between experiment and simulation observations can generically be formulated as:

$$
\mathbf{q}(t)+\boldsymbol{\eta}(t)=\mathcal{G}(t)+\boldsymbol{\epsilon}(t)
$$

On the left-hand side is the experimental observation of the "truth" $\mathbf{q}$ (to reuse the term employed in [19]), polluted by some necessary observation errors $\boldsymbol{\eta}$. On the right-hand side is the output of the numerical model $\mathcal{G}$ along with the simulation errors gathered in $\boldsymbol{\epsilon}$. Hence, $\boldsymbol{\epsilon}$ accounts for the effects of a possible lack of physics in $\mathcal{G}$ as well as for any other reason why the output of $\mathcal{G}$ does not fit the experimental observations, such as numerical errors due to digitized space and time integration procedures for instance.

Here, we focus on acceleration and displacement time histories as quantities of interest. Accelerations provide insight in the resisting forces developed in the structure; displacements are, as mentioned in the introduction, often used as indicators of the structural response that can be mapped to performance levels. Besides, we do not explicitly account for any measurement error. Consequently, the right-hand side in equation (9) takes the form $\mathbf{q}+\boldsymbol{\eta}=\{\mathbf{y} \ddot{\mathbf{y}}\}^{T}$. On another hand, we set $\mathcal{G}=\mathcal{H} \star \mathcal{D}$, where $\star$ denotes the dual actions of model $\mathcal{H}$ (inertia 
along with inelastic structural resisting forces) and model $\mathcal{D}$ (discrepancy forces). We have $\mathcal{G}(t)=\{\mathbf{u} \ddot{\mathbf{u}}\}^{T}$ where $\mathbf{u}$ and $\ddot{\mathbf{u}}$ result from either solving $\mathbf{R}=\mathbf{0}$ when $\mathcal{D}$ is defined such that $\mathbf{F}^{\text {dis }}=\mathbf{C} \dot{\mathbf{u}}$ (equation (5)), or from solving $\tilde{\mathbf{R}}=\mathbf{0}$ when $\mathbf{F}^{\text {dis }}$ is not given any particular form (equation (7)).

Discrepancy forces are computed as the solution of the inverse problem $\tilde{\mathbf{R}}(\mathbf{y}, \ddot{\mathbf{y}})=$ $\mathbf{0}$, which according to equation (7) leads to:

$$
\mathbf{F}^{\text {dis }}=\mathbf{F}^{e x t}-\mathbf{M} \ddot{\mathbf{y}}-\mathbf{F}^{\text {hys }}(\mathbf{y})
$$

In other words: the external forces along with the displacements $\mathbf{y}(t)$ and accelerations $\ddot{\mathbf{y}}(t)$ recorded during shaking table test are imposed to the system, which yields $\tilde{\mathbf{R}}=\mathbf{0}$ because $\mathbf{y}(t)$ and $\ddot{\mathbf{y}}(t)$ are the "true" displacements and accelerations; then $\mathbf{F}^{\text {dis }}$ can be directly computed with equation (10). Discrepancy forces are then defined as the forces needed in the system to satisfy the balance equation without uncertain viscous damping forces. $\mathbf{M}$ and $\mathbf{F}^{\text {hys }}$ are computed from a FE model, $\mathbf{F}^{e x t}$ contains the static forces and the seismic forces computed from $\mathbf{M}$ and the acceleration of the shaking table $\ddot{u}_{g}$ recorded during the test.

Note that in the case $\mathbf{F}^{\text {dis }}$ is defined as a particular model, for instance as Rayleigh damping forces $\left(\mathbf{F}^{\text {dis }}=\mathbf{C} \dot{\mathbf{y}}\right.$ with $\left.\mathbf{C}=\alpha \mathbf{M}+\beta \mathbf{K}\right)$, there is no guarantee the problem $\tilde{\mathbf{R}}(\mathbf{y}, \ddot{\mathbf{y}})=\mathbf{0}$ has a solution. This would not mean that the model $\mathcal{D}$ for building $\mathbf{F}^{\text {dis }}$ (Rayleigh damping) intrinsically is poor, but it would mean that the dual action of model $\mathcal{H}$ and Rayleigh damping model is not suitable for accurately simulating the experimentally observed displacement and acceleration time histories.

More specifically, suppose one has a $N$-degree-of-freedom (DOF) FE model of a structure that is tested on a shaking table. Then, suppose both the relative displacement and relative acceleration time histories are recorded during the experimental test at $N^{e} \leq N$ DOFs of the FE mesh of the structure and gathered in vectors $\mathbf{y}^{e}(t)$ and $\ddot{\mathbf{y}}^{e}(t)$ of size $N^{e}$. Reorganizing the DOFs order so that the $N^{e}$ free DOFs that are monitored during the experimental test as well as the $N^{b}$ DOFs controlled by structural boundary conditions are gathered, it comes:

$$
\left(\begin{array}{l}
\mathbf{F}^{d i s, e} \\
\mathbf{F}^{d i s, f} \\
\mathbf{F}^{d i s, b}
\end{array}\right):=\left(\begin{array}{l}
\mathbf{F}^{e x t, e} \\
\mathbf{F}^{e x t, f} \\
\mathbf{F}^{e x t, b}
\end{array}\right)-\mathbf{M}\left(\begin{array}{l}
\ddot{\mathbf{y}}^{e} \\
\ddot{\mathbf{y}}^{f} \\
\ddot{\mathbf{y}}^{b}
\end{array}\right)-\left(\begin{array}{l}
\mathbf{F}^{h y s, e}\left(\mathbf{y}^{e}, \mathbf{y}^{f}, \mathbf{y}^{b}\right) \\
\mathbf{F}^{h y s, f}\left(\mathbf{y}^{e}, \mathbf{y}^{f}, \mathbf{y}^{b}\right) \\
\mathbf{F}^{h y s, b}\left(\mathbf{y}^{e}, \mathbf{y}^{f}, \mathbf{y}^{b}\right)
\end{array}\right) .
$$

In an ideal situation, the displacement and acceleration time histories have been recorded for all the DOFs of the FE mesh. Then, $\mathbf{F}^{\text {hys }}\left(\mathbf{y}^{e}, \mathbf{y}^{b}\right)$ can be computed in a quasi-static nonlinear structural analysis, where the structure is loaded by the displacements $\mathbf{y}^{e}$, to yield the discrepancy forces. However, in practice, there is usually not such an amount of data recorded and there are $N^{f}$ DOFs not monitored during shaking table test. Some assumptions have to be made to cope with this lack of knowledge on the experimental response of the structure to compute $\mathbf{F}^{\text {dis }}$. 
The discrepancy forces provide information on the capability of the structural model to represent the experimental response of the structure or, in other words, the forces that should be added in the system to account for the mechanisms either not or poorly represented by the inelastic structural response $\mathbf{F}^{\text {hys }}$.

2.4. Errors sources. Equation (9) actually involves the $N^{e}$ DOFs where experimental data is available for both displacement and acceleration time histories $\left(\mathbf{y}^{e}(t)\right.$ and $\left.\ddot{\mathbf{y}}^{e}(t)\right)$. On the other hand, equation (7) involves the other $N^{f}$ DOFs defined in the finite element model. In turn, only the elements of the discrepancy forces vector pertaining to the $N^{e}$ controlled DOFs can be identified. Compromises based on expert judgement then have to be made to define the $N^{f}$ other discrepancy forces. Then, solving $\tilde{\mathbf{R}}(\mathbf{u}, \ddot{\mathbf{u}})=\mathbf{0}$ does not necessarily yield $\left\{\mathbf{u}^{e}(t) \ddot{\mathbf{u}}^{e}(t)\right\}=\left\{\mathbf{y}^{e}(t) \ddot{\mathbf{y}}^{e}(t)\right\}$, especially in case some DOFs that significantly contribute to the overall structural response have not been monitored during shaking table test and can thus not be controlled when solving $\tilde{\mathbf{R}}=\mathbf{0}$.

It is also possible that seismic analysis run with discrepancy forces computed as in equation (11) instead of damping forces does not exactly yield the displacements and accelerations recorded during the experimental test. This comes from the asymmetrical numerical treatment of the inverse and direct problems. Indeed, on the one hand, inverse problem $\tilde{\mathbf{R}}\left(\mathbf{y}^{e}, \ddot{\mathbf{y}}^{e}\right)=\mathbf{0}$ is quasi-statically solved to compute $\mathbf{F}^{h y s, e}(t): \mathbf{y}^{e}(t)$ is imposed step by step, without dynamic effects, and the resisting forces are stored. Whereas, on the other hand, the direct problem $\tilde{\mathbf{R}}(\mathbf{u}, \ddot{\mathbf{u}})=\mathbf{0}$ is solved dynamically. Consequently, errors due to the time integration algorithm are only generated when solving the direct problem.

Finally, it is worth stressing that $\mathcal{G}$ somehow acts like a black box. Suppose the dual action of a structural model $\mathcal{H}$ with a Rayleigh damping model allows for accurately simulating $\{\mathbf{u}(t) \ddot{\mathbf{u}}(t)\}=\{\mathbf{y}(t) \ddot{\mathbf{y}}(t)\}$. This only means $\mathcal{G}$ is a valid model for predicting the quantities of interest (displacements and accelerations). It however in any way means that the structural model $\mathcal{H}$ or the discrepancy forces model (Rayleigh damping), independently taken, are valid models. As a consequence, there is no guarantee the variables used in model $\mathcal{H}$ to describe the state of the system are accurate. For instance, $\mathcal{G}$ being a valid model does not imply the plastic deformations in steel rebars are accurately simulated.

\section{Shaking TABle tests}

3.1. Description of the structure. In this work, experimental data recorded during the shaking-table test of a ductile moment-resisting reinforced concrete frame is used [7]. The structure is depicted in figure 1. It was designed at a reduced scale of $1 / 2$ according to the provisions of the National Building Code of Canada and of the Canadian concrete standard. The structure was assumed to be ductile, that is the design base shear was computed with a reduction factor $R=4$. The frame is fixed in a stiff base beam that is clamped on the shaking table. 
No rocking has been reported during the test. Four concrete blocks were used to simulate concentrated gravity loads in every beam span; they were designed so that their center of gravity coincide with the center of gravity of the supporting beam. These latter additional masses induced service cracks. The total weight of the frame is about $100 \mathrm{kN}$, its fundamental period $T_{1}=0.28 \mathrm{~s}$ was measured preliminary to the seismic excitation, with the additional concrete blocks. Mode 1 is preponderant in the sense that it accounts for more than $90 \%$ of the total mass. The detailed presentation of the design assumptions and parameters can be found in $[7]$.

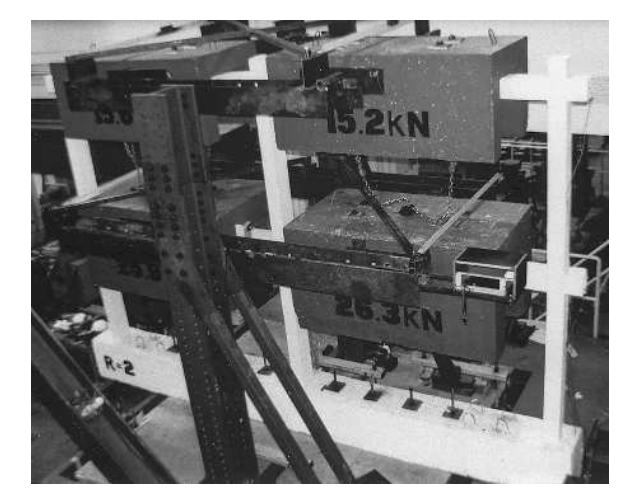

Figure 1. RC frame structure tested on the shaking table at École Polytechnique Montreal. The frame is 5 -meter wide $(2 \times 2.5 \mathrm{~m})$ and 3 -meter high $(2 \times 1.5 \mathrm{~m})$. Every beam supports additional masses to account for service static loads. The system is symmetric.

3.2. Loading. The structure is subjected to two types of loading: vertical static loading due to the dead load of the frame along with the additional masses, and horizontal dynamic forces induced by the seismic acceleration time history imposed on its base.

The N04W component of the ground motion recorded in Olympia, Washington on April 13, 1949 was selected for the test program and scaled to a peak ground acceleration PGA $=0.21 \mathrm{~g}$. The feedback record of the acceleration measured on the shaking table during the test is shown in figure 2 and the corresponding elastic response spectrum with $5 \%$ viscous damping ratio in figure 3 . This is the ground motion time history actually imposed on the base of the structure. So, it accounts for the effects of possible friction in the shaking table and of other energy dissipation mechanisms in the experimental contrivance that could be activated and that are external to the structure (interaction with the surrounding air is neglected and there is no interaction observed between the test structure and the system to prevent out-of-plane bending). 


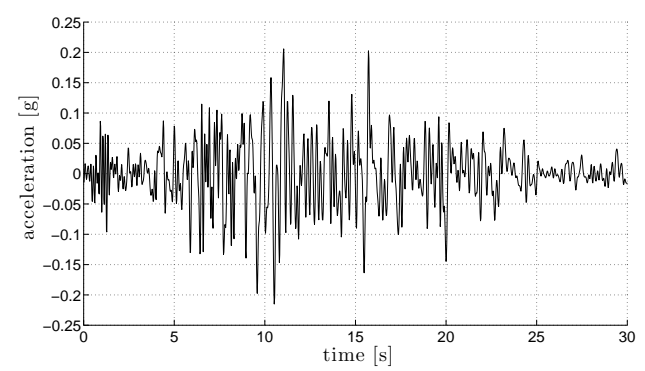

FiguRE 2. Acceleration time history recorded on the base of the frame during the test.

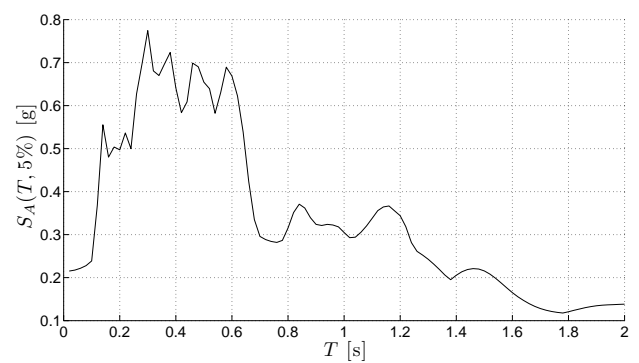

FIGURE 3. Elastic response spectrum to the seismic motion with a critical viscous damping ratio of $5 \%$ (pseudo-acceleration with respect to period).

3.3. Data recorded during the test. During the experimental test, the following acceleration and displacement time histories have been recorded (node numbers are consistent with figure 4):

- the horizontal absolute displacement of the shaking table (precision $\pm 0.9 \mathrm{~mm}$ ) and at nodes 4 and 11 (precision $\pm 0.3 \mathrm{~mm}$ ), as well as the absolute vertical displacement at nodes 5, 6, 8 and 9 (precision $\pm 0.2 \mathrm{~mm}$ );

- the horizontal absolute acceleration of the shaking table and at nodes 4 and 11 (precision $\pm 0.005 \mathrm{~g}$ ).

Besides, local deformation time histories in several longitudinal rebars were recorded. In what follows, only the horizontal displacements and accelerations are used.

\section{Two inelastic structural models: $\mathcal{H}_{1}$ And $\mathcal{H}_{2}$}

All the numerical simulations are performed with the finite element computer program FEAP [22] where the various elements and material behavior laws have been implemented.

4.1. Two finite element models: $\mathcal{H}_{1}$ and $\mathcal{H}_{2}$. Two different finite element models of the tested frame are developed, as illustrated in figure 4 . The frame 
remained in its initial plane during the shaking table test and it is thus modeled as a 2D structure. Both models have the same geometry and material properties, they differ one from another with respect to the way beam-to-column connections are treated. For both models, end zones in elements 8, 11, 14 and 17 are modeled as very stiff comparing to current beam sections stiffness. This aims at representing the stiffening effects of the steel plates added on the beams as part of the system designed for clamping together the additional masses and the frame. Otherwise, for model $\mathcal{H}_{1}$, no particular treatment of the elements connections is introduced. For model $\mathcal{H}_{2}$ however, the beam-to-column inelastic joints elements shown in figure 6 are used.

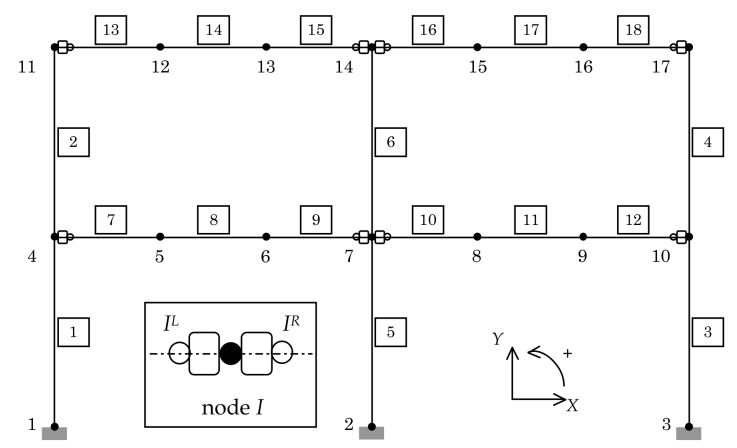

FiguRE 4. Finite element meshes for structural models $\mathcal{H}_{1}$ (black nodes only) and $\mathcal{H}_{2}$ (black and white nodes connected through inelastic joints).

4.2. Inertia forces. It is reported in [14] that the experimentally measured mass per unit volume of frame is $\rho=3200 \mathrm{~kg} \cdot \mathrm{m}^{-3}$. This supposedly uniformly distributed mass is concentrated on the nearest nodes all over the frame. Additional mass on element 8 is concentrated at nodes 5 and 6 , and analogously for the three other beams 11, 14 and 17. For model $\mathcal{H}_{2}$, the additional nodes (white nodes in figure 4) are considered as massless. Rotational DOFs are assumed as massless too, whereas same mass is assigned to both vertical and horizontal DOFs. Accordingly, we compute the inertia forces with the following masses: $m_{4}=m_{10}=156.4 \mathrm{~kg}$, $m_{5}=m_{6}=m_{8}=m_{9}=1434.0 \mathrm{~kg}, m_{7}=224.5 \mathrm{~kg}, m_{11}=m_{17}=90.5 \mathrm{~kg}$, $m_{12}=m_{13}=m_{15}=m_{16}=861.2 \mathrm{~kg}, m_{14}=136.8 \mathrm{~kg}$.

4.3. Inelastic beam and column elements. Beams and columns are modeled using fiber elements [4] with Euler-Bernoulli kinematics and displacement-based numerical implementation. Control cross sections along the frame element axis coincide with the position of two Gauss-type numerical integration points. Because this is a 2D-model, these control sections are divided into concrete layers, and not fibers as suggested by its denomination. The steel longitudinal rebars are accurately positioned in the elements cross sections. Uniaxial material behavior 
law is then assigned to every concrete layer and steel fiber. Steel reinforcing stirrups are not explicitly accounted for in the model. However, they are expected to confine concrete and, consequently, confined and unconfined concrete layers with different behavior laws are implemented in the model, as illustrated in figure 5 .

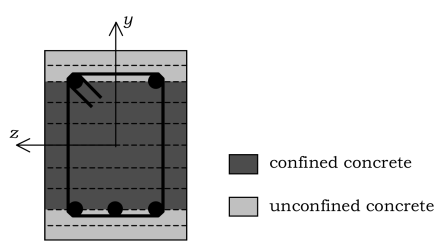

FiguRE 5. Frame element cross section. Sections are divided into confined and unconfined concrete layers according to whether it is inside or outside the steel reinforcement stirrups (in the $y$ direction). Longitudinal steel rebars cross sections are explicitly introduced in the model description, not the stirrups. Dashed lines represent frontiers between layers.

To get a structural response that is independent of the number of layers in the elements, it has been observed that the following cross-section discretization in all the frame elements is suitable: 4 unconfined concrete layers (2 at the top and 2 at the bottom) and 6 confined concrete layers, as represented in figure 5. Besides, second-order P- $\Delta$ effect is accounted for in every frame columns.

4.4. Inelastic beam-column joint element. For assessing structural performance of reinforced concrete frames, beam-column joint elements have been developed and implemented in finite element procedures for modeling reinforced concrete moment-resisting frames, whether they are ductile or not $[1,17]$. The joint elements used in these two papers account for the main inelastic mechanisms that determine beam-column behavior: that is shear cracking, longitudinal rebars yielding and anchorage failure. In the present work, we use a simple model for representing the inelastic response of beam-column joints, very much in the spirit of the nonlinear rotational springs initially effectively used in [8] for modeling the same frame as in this present work. Indeed, the purpose here is not to test the capability of elements to effectively represent complex observed structural behaviors, but to illustrate how using different structural models affects the discrepancy forces.

Accordingly, the basic model illustrated in figure 6 as been developed and numerically implemented to limit resisting bending moment at beam-to-column connections. The joint acts like a rigid body along horizontal and vertical directions. It has however finite stiffness in rotation represented by two bars which develop a force $F$ according to an inelastic moment-rotation behavior law. These joint bars are extensions of the longitudinal rebars in the adjacent beam: they have the same position in the section and the same geometry. 


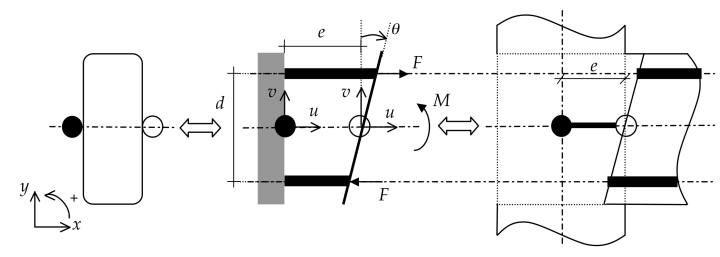

Figure 6. Beam-to-column element.

4.5. Material behavior laws. Although results of uniaxial test carried out to identify the actual properties of the steel and concrete materials the frame structure is built of are reported in $[7,14]$, there is too few experimental data available for accurately identifying steel and concrete uniaxial cyclic responses. As a consequence, the material constitutive laws detailed below are thought as reasonable approximations of the actual behaviors. All the material models considered in this work have been developed using some of the ingredients of the more general model presented in [12].

4.5.1. Longitudinal steel rebars. Mean Young modulus $E_{s}=224.6 \mathrm{GPa}$, mean yield stress $\sigma_{y}=438 \mathrm{MPa}$ and mean ultimate stress $\sigma_{u}=601 \mathrm{MPa}$ have been obtained from uniaxial tests on longitudinal steel rebars. The steel model implemented is shown in figure 7 .

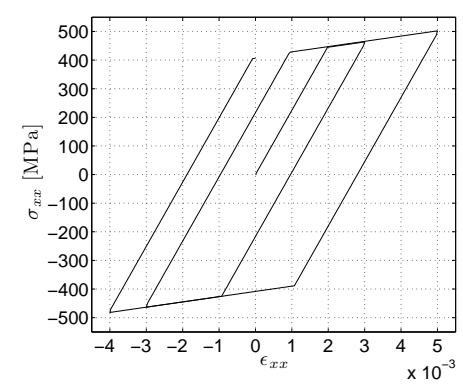

FIgURE 7. Steel longitudinal rebar constitutive law.

4.5.2. Concrete. Compression tests have been carried out on unconfined concrete specimens, leading to mean Young modulus of $E_{c}=27.5 \mathrm{GPa}$ and ultimate compression stress $\sigma_{c}^{\prime}=26.2 \mathrm{MPa}$. Concerning the behavior of concrete confined inside the stirrups, no experimental data is available and it is modeled with expected larger ultimate stress and additional ductility than for unconfined concrete. Concrete models implemented are shown in figure 8. 


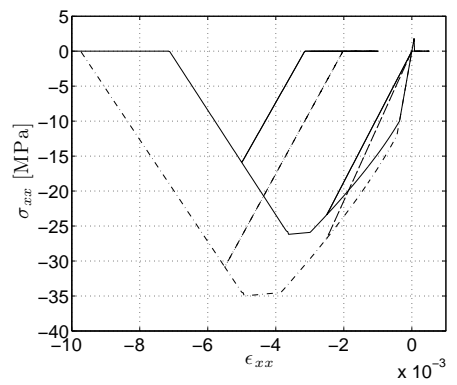

FiguRE 8. Concrete behavior laws: unconfined (plain line) and confined (dashed line) concrete.

4.5.3. Joint bars. As illustrated in figure 6, beam-column joint elements momentrotation response is modeled by upper and lower bars. The purpose of these joints is to limit the resisting bending moment in the frame elements. Accordingly, inelastic behavior illustrated in figure 9 is assigned to the joint bars. This behavior is symmetric and parameterized by an elastic stiffness $E_{j}$ along with a limit stress $\sigma_{j}$. Post-yielding stiffness is fixed at less than $0.1 E_{j}$. Assuming equal areas $A$ for the upper and lower bars cross sections, the limit moment is computed as $M_{j}=A \sigma_{j} d$.

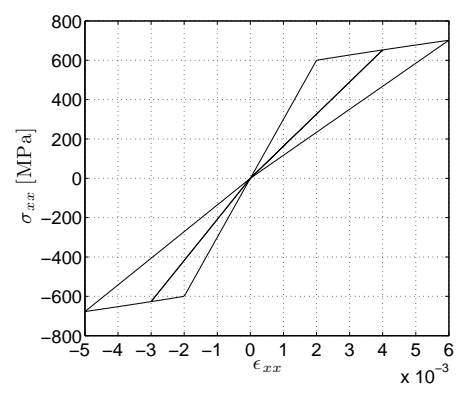

FiguRE 9. Beam-to-column joint behavior law. Illustration with $E_{j}=300 \mathrm{GPa}, \sigma_{j}=600 \mathrm{MPa}$.

\section{Model $\mathcal{G}_{1}=\mathcal{H}_{1} \star \mathcal{D}_{1}$}

In this section, we identify a model of discrepancy forces $\mathcal{D}_{1}$. Only an approximation of the discrepancy forces can be provided because experimental data are missing to calculate the complete discrepancy forces vector. Given the available data, it is assumed that the horizontal displacements and accelerations at each node from node 4 to node 10 (see figure 4 ) are equal, and so for the $2^{\text {nd }}$-level beam (nodes 11 to 17). This assumption is justified by the fact that the dynamic 
response of the frame is dominated by its $1^{\text {st }}$ mode and that this preponderant mode is an overall lateral bending mode.

5.1. Inelastic response to static loading. The elastic fundamental period of the frame is unknown, but this period after the additional masses are applied has been experimentally measured through free vibration tests: $T_{1}^{e x p}=0.28 \mathrm{~s}$. We use this information to check whether model $\mathcal{H}_{1}$ accurately represents structural mass and stiffness distribution in the initial state before seismic loading.

Due to the possible inelastic response of the structure, static loading is first imposed as vertical nodal forces during a step by step numerical process. Then, numerical free vibration test is carried out and it is observed that $T_{1}^{\mathcal{H}_{1}}=0.23 \mathrm{~s}$ before static loading is applied and $T_{1}^{\mathcal{H}_{1}}=0.29 \mathrm{~s}$ after. This good agreement with the experimental measure shows the capability of model $\mathcal{H}_{1}$ to accurately represent the stiffness and mass distributions over the structure loaded by the static weights.

5.2. Discrepancy forces model $\mathcal{D}_{1}$. The recorded time histories during the shaking table test consist in the horizontal relative displacement $\left(\mathbf{y}^{e}\right)$ and relative acceleration $\left(\ddot{\mathbf{y}}^{e}\right)$ at the $1^{\text {st }}$ and $2^{\text {nd }}$ levels of the frame, along with the shaking table acceleration $\left(\ddot{u}_{g}\right)$. 'Relative' is to be understood as 'relative to the displacement and acceleration at the base of the frame'. Displacements induced by static loading (additional masses fixed on the beams before the seismic motion) have not been recorded. To account for the contribution of static loading to the discrepancy forces, these displacements are approximated performing quasi-static numerical analysis and storing the displacements pertaining to the $N^{e}$ DOFs monitored during shaking table test, hereafter referred to as $\mathbf{u}^{\text {sta,e }}$. We assume that there is no dynamic effects generated while static loading is added on the frame $\left(\ddot{\mathbf{u}}^{\text {sta,e }}=\mathbf{0}\right)$.

Accordingly, discrepancy forces are computed in the following steps:

(1) A quasi-static analysis is run with the static forces imposed to the structure and the induced horizontal displacements $\mathbf{u}^{\text {sta,e }}$ are stored.

(2) Resisting forces $\mathbf{F}^{\text {hys }}$, that is the response of model $\mathcal{H}_{1}$, are computed solving the following quasi-static nonlinear problem:

$$
\left(\begin{array}{l}
\mathbf{0} \\
\mathbf{0} \\
\mathbf{0}
\end{array}\right):=\left(\begin{array}{c}
\mathbf{0} \\
\mathbf{F}^{s t a, f} \\
\mathbf{F}^{\text {sta,b }}
\end{array}\right)+\left(\begin{array}{c}
\mathbf{V}^{e} \\
\mathbf{0} \\
\mathbf{0}
\end{array}\right)-\left(\begin{array}{l}
\mathbf{F}^{h y s, e}\left(\mathbf{u}^{s t a, e}+\mathbf{y}^{e}, \mathbf{y}^{f}, \mathbf{0}\right) \\
\mathbf{F}^{h y s, f}\left(\mathbf{u}^{s t a, e}+\mathbf{y}^{e}, \mathbf{y}^{f}, \mathbf{0}\right) \\
\mathbf{F}^{h y s, b}\left(\mathbf{u}^{s t a, e}+\mathbf{y}^{e}, \mathbf{y}^{f}, \mathbf{0}\right)
\end{array}\right),
$$

where the displacements $\mathbf{u}^{\text {sta,e }}$ along with $\mathbf{y}^{e}$ are imposed to the structure. $\mathbf{V}^{e}$ is the vector containing the reaction forces at the DOFs where these latter displacements are imposed, and $\mathbf{F}^{\text {sta }}$ is the static part of the external loading. Static loading (vertical forces) is imposed within the same time period than $\mathbf{u}^{\text {sta,e }}$ and then kept constant while $\mathbf{y}^{e}$ is applied to the structure. 
(3) Due to a lack of recorded data, we assume that dynamic effects for the vertical and rotational DOFs can be neglected, that is $\ddot{\mathbf{y}}^{f}=\mathbf{0}$. Besides, there only are dynamic external loading forces imposed to the $N^{e}$ DOFs monitored during shaking table test, so that there are only static forces applied at the other nodes: $\mathbf{F}^{e x t, f / b}=\mathbf{F}^{s t a, f / b}$. Finally, using the experimentally recorded accelerations $\ddot{\mathbf{y}}^{e}$ and $\ddot{u}_{g}$, the discrepancy forces $\mathbf{F}^{\text {dis }}$ are computed introducing equations (12) in equations (11):

$$
\left(\begin{array}{l}
\mathbf{F}^{d i s, e} \\
\mathbf{F}^{d i s, f} \\
\mathbf{F}^{d i s, b}
\end{array}\right):=\left(\begin{array}{c}
-\mathbf{M}^{e} \mathbf{1}^{e} \\
\mathbf{F}^{s t a, f} \\
\mathbf{F}^{s t a, b}
\end{array}\right)-\left(\begin{array}{c}
\mathbf{M}^{e} \ddot{\mathbf{y}}^{e} \\
\mathbf{0} \\
\mathbf{0}
\end{array}\right)-\left(\begin{array}{c}
\mathbf{V}^{e} \\
\mathbf{F}^{s t a, f} \\
\mathbf{F}^{s t a, b}
\end{array}\right),
$$

where $\mathbf{1}^{e}$ is the unit vector of size $N^{e}$. Accordingly, $\mathbf{F}^{d i s, f}=\mathbf{F}^{d i s, b}=\mathbf{0}$ and

$$
\mathbf{F}^{d i s, e}=-\mathbf{M}^{e}\left(\ddot{\mathbf{y}}^{e}+\mathbf{1}^{e} \ddot{u}_{g}\right)-\mathbf{V}^{e},
$$

with, according to equation (12), $\mathbf{V}^{e}=\mathbf{F}^{\text {hys,e }} . \mathbf{F}^{\text {sei,e }}=-\mathbf{M}^{e} \mathbf{1}^{e} \ddot{u}_{g}$ is the seismic forces vector and $\mathbf{F}^{i n e, e}=\mathbf{M}^{e} \ddot{\mathbf{y}}^{e}$ the inertia forces vector.

$\mathbf{F}^{\text {dis,e }}$ reflects the capacity of the inelastic structural model to represent the behavior that has been experimentally observed during shaking table test: if the structural model were capable of perfectly representing the experimental response of the analyzed frame, discrepancy forces would be null for each DOF. Typical seismic and inertia forces as well as discrepancy and hysteretic forces time histories computed following this method are shown in figures 10 and 11. Discrepancy and hysteretic forces looks like they are symmetric with respect to the $x$-axis. In fact, according to equation (14), they would be symmetric if the dynamic forces were null. Seismic and inertia forces are however not null but very small comparing with the hysteretic forces (compare figures 10 and 11).

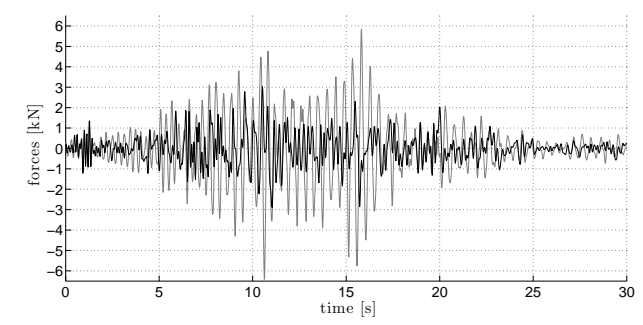

FiguRE 10. Seismic (black) and inertia forces (grey) at node 5. The mass distribution for model $\mathcal{H}_{1}$ being the same as for model $\mathcal{H}_{2}$, seismic and inertia forces are identical for both models. Seismic and inertia forces at any other node are less or equal to those shown here at node 5 . 

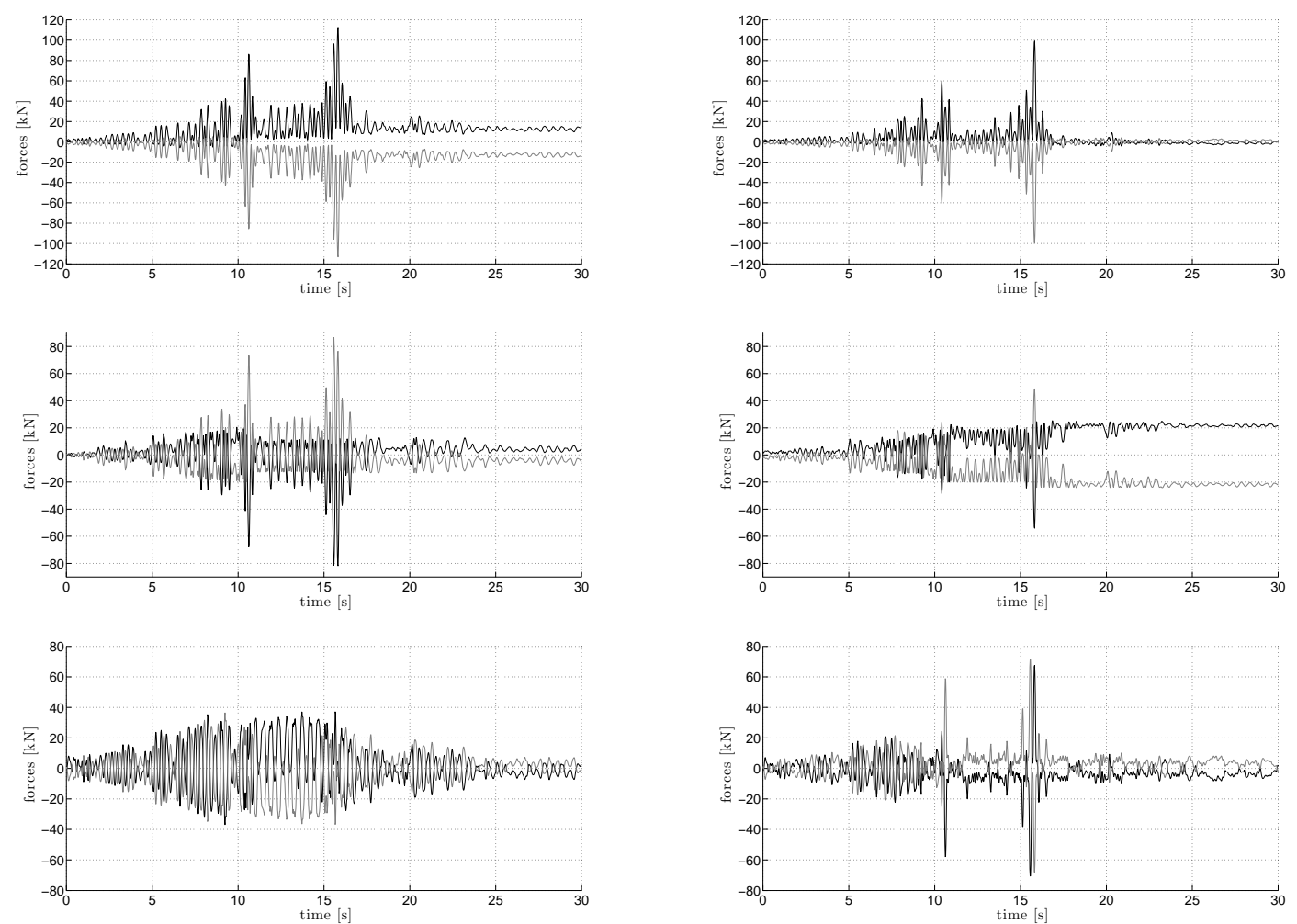

Figure 11. Model $\mathcal{D}_{1}$ [left] and model $\mathcal{D}_{2}$ [right]. Forces at nodes 4 [top], 5 [center], and 7 [bottom]: discrepancy forces (black), resisting forces developed by inelastic structural models (grey).

5.3. Seismic response of $\mathcal{G}_{1}$. Usual seismic analysis is then run, except without Rayleigh damping forces (commonly added in practice) and with the previously identified discrepancy forces imposed to the structure, the same way external loading is. This means the direct problem $\tilde{\mathbf{R}}(\mathbf{u}, \ddot{\mathbf{u}})=\mathbf{0}$ is solved to check whether or not the computed displacements and accelerations $\mathbf{u}^{e}$ and $\ddot{\mathbf{u}}^{e}$ fit the experimental data $\mathbf{y}^{e}$ and $\ddot{\mathbf{y}}^{e}$. The HHT- $\alpha$ method [11] implemented in FEAP with $\alpha=0.65$, $\beta=0.5$ and $\gamma=1$ is used and a time step of $5 \mathrm{~ms}$; the value for $\alpha$ has been found to be adequate for accurately simulating the maximum displacements.

The comparison of the numerical response $\mathcal{G}(t)$ with the experimental data is shown in figure 12. The horizontal displacements are well simulated at both levels, except for the first 7 or $8 \mathrm{~s}$ when neither the amplitude nor the phase of the signals match. Considering the accelerations time histories, the numerical response is in good accordance with the experimental data for the $2^{\text {nd }}$ level, especially between 8 and $20 \mathrm{~s}$, as for the displacements. For the $1^{\text {sd }}$ level, the numerical response does not fit as good the experimental recording. One can then conclude that model 

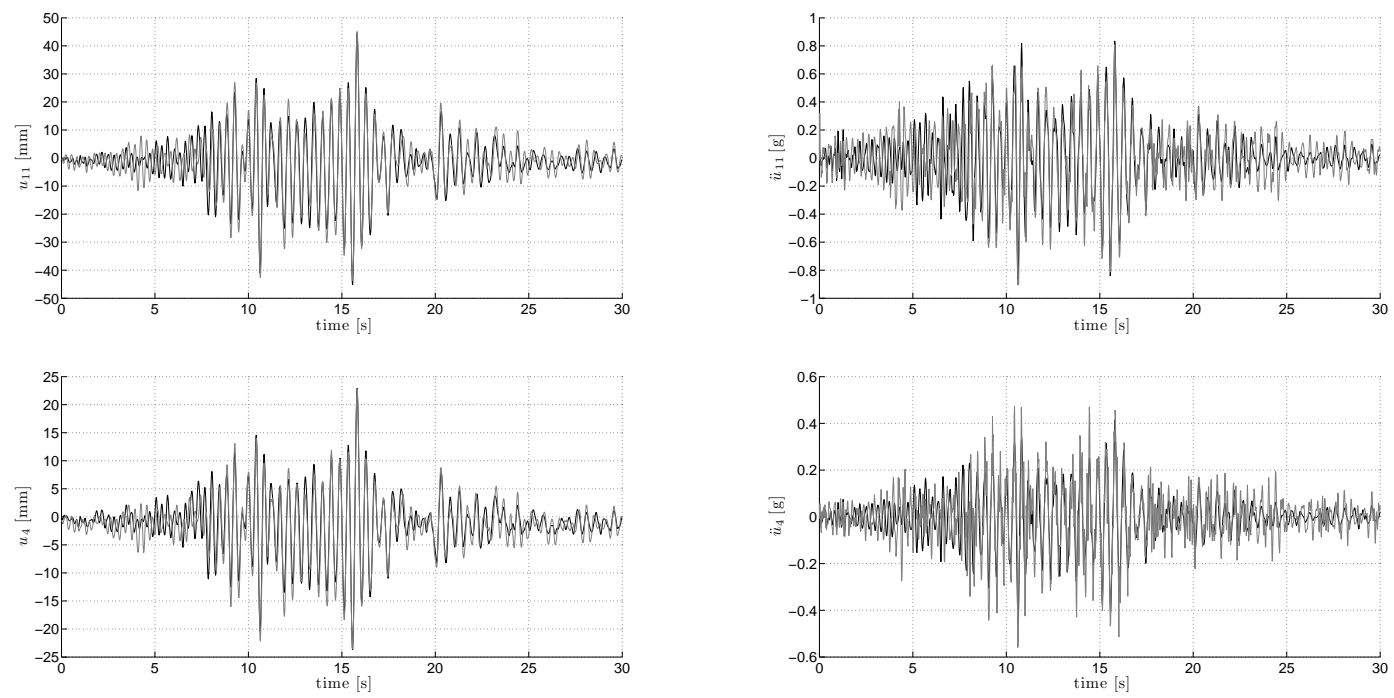

FiguRE 12. [top] $2^{\text {nd }}$-level relative displacement [left] and relative acceleration [right] time histories; [bottom] $1^{\text {st }}$-level relative displacement [left] and relative acceleration [right] time histories. Experimental data (black) and numerical simulation with model $\mathcal{G}_{1}$ (grey).

$\mathcal{G}(t)$ provides a reasonably accurate model for predicting horizontal displacements and accelerations in the frame.

5.4. A critical look into model $\mathcal{H}_{1}$. As illustrated in figure 11 [left], the discrepancy forces are very large comparing with the seismic and inertia forces. This sheds light on the fact that the model $\mathcal{H}_{1}$ is unable to accurately represent the hysteretic forces actually developed in the frame elements during shaking table test. If model $\mathcal{H}_{1}$ were an accurate model, the hysteretic forces developed due to the imposed displacements $\mathbf{y}^{e}$ would approximately satisfy the force equilibrium equations without the need to add discrepancy forces to satisfy the equilibrium equations. However here, large discrepancy forces are required to balance the large hysteretic forces.

Consequently, we developed another inelastic structural model $\mathcal{H}_{2}$ which is analogous to $\mathcal{H}_{1}$, except at the beam-to-column connections (see figure 4 ) where inelastic behavior limiting the resisting moments is accounted for.

\section{MODEL $\mathcal{G}_{2}=\mathcal{H}_{2} \star \mathcal{D}_{2}$}

6.1. Parameterization of the model. The joint elements are parameterized by the elastic modulus $E_{j}$ and the limit stress $\sigma_{j}$ (post-yielding slope is set to less than $\left.0.1 E_{j}\right) . E_{j}$ is identified so that the fundamental period of the frame after dead load is applied approaches the experimental value $T_{1}^{\exp }=0.28 \mathrm{~s}$ : setting 
$E_{j}=300 \mathrm{GPa}$ yields to $T_{1}^{\mathcal{H}_{2}}=0.29 \mathrm{~s}$ for the frame with static loading and to $T_{1}^{\mathcal{H}_{2}}=0.25 \mathrm{~s}$ before static loading. Then, $\sigma_{j}$ is calculated so that the bending moment at beam-to-column connections is limited to $\pm \bar{M}_{z}$ as the appearance of large discrepancy forces are detected in model $\mathcal{D}_{1}$. We set $\bar{M}_{z}=13 \mathrm{kN}$.m, which corresponds to $\sigma_{j}=600 \mathrm{MPa}$, and here is how we chose this value: i) According to the time history of the discrepancy forces for model $\mathcal{D}_{1}$ shown in figure 11 [left], one can observe that the discrepancy forces increase around $t=8 \mathrm{~s}$; ii) Then, the time history of the bending moment at node 7 in element 9, plotted in figure 13, shows that, around $t=8 \mathrm{~s}$, bending moment exceeds $M_{z}=-13 \mathrm{kN} . \mathrm{m}$; iii) In the attempt to limit the discrepancy forces, we thus limit the bending moment to $\bar{M}_{z}=13 \mathrm{kN} . \mathrm{m}$. The bending moment could have been monitored at another beam-to-column connection and another value of $\bar{M}_{z}$ could have been observed. However, our purpose here is only to develop a structural model $\mathcal{H}_{2}$ that is different from previous structural model $\mathcal{H}_{1}$, and not to rigorously identify the beam-tocolumn joint parameters that would lead to the best representation of the frame. This would require much more advanced identification procedures that are out of the scope of this work.

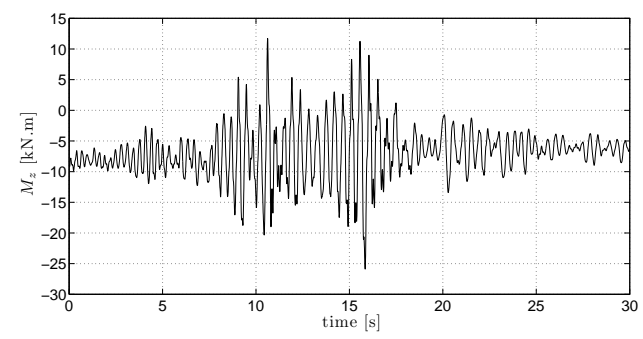

FiguRE 13. Resisting bending moment time history at node 7 in element 9 simulated running model $\mathcal{G}_{1}$. Bending moment is not null initially because of preceding static loading.

6.2. Discrepancy forces model $\mathcal{D}_{2}$. The discrepancy forces for model $\mathcal{D}_{2}$ are calculated as for model $\mathcal{D}_{1}$. Figure 11 [right] shows the same quantities for model $\mathcal{D}_{2}$ as figure 11 [left] for model $\mathcal{D}_{1}$. It can be observed that the discrepancy forces are overall smaller for model $\mathcal{D}_{2}$, except in two cases: i) at node 5 for $t>16 \mathrm{~s}$ and ii) at node 7 where the resisting and discrepancy forces drop around $t=11 \mathrm{~s}$ and $t=16 \mathrm{~s}$ are larger than for model $\mathcal{D}_{1}$.

6.3. Seismic response of $\mathcal{G}_{2}$. We first show in figure 14 the bending moment time history at node $7^{L}$ to check the efficiency of the inelastic joints for limiting the bending moment between $\pm 13 \mathrm{kN}$.m.

As for model $\mathcal{G}_{1}$, the direct problem is solved as a usual seismic analysis except with the discrepancy forces from model $\mathcal{D}_{2}$ and not, as in common practice, from a Rayleigh damping model. Again, simulated displacements and accelerations are 


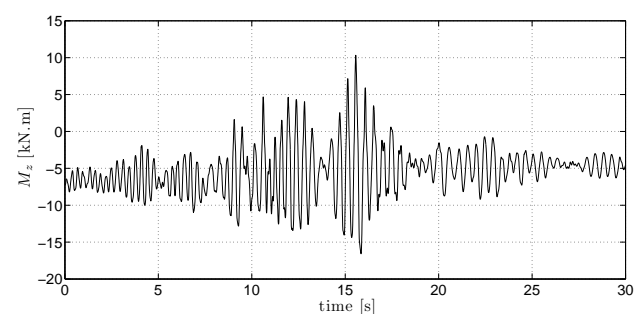

FiguRE 14. Resisting bending moment time history at node $7^{L}$ (element 9) simulated running model $\mathcal{G}_{2}$. Bending moment is not null initially because of preceding static loading.

compared with the experimental data (see figure 15). Displacements at both levels are fairly good simulated, while larger errors are observed for the accelerations. As an overall remark, experimental data are not as good represented as with model $\mathcal{G}_{1}$. Note that model $\mathcal{G}_{2}$ not performing as good as model $\mathcal{G}_{1}$ is not in contradiction with the fact that model $\mathcal{H}_{2}$ is more elaborate than model $\mathcal{H}_{1}$. It only means that the discrepancy forces for model $\mathcal{G}_{2}$ are not as accurately computed as for model $\mathcal{G}_{1}$. This can be explained by the fact that there are other DOFs than the horizontal ones (the rotational DOFs) that significantly contribute to the structural response for model $\mathcal{G}_{2}$. For model $\mathcal{G}_{1}$ however, beam-to-column joints behave as rigid bodies and do not contribute to the overall seismic energy absorption. Also, the abrupt stiffness changes in the joint elements could generate some artifacts, which the acceleration time history could be especially affected by. The sensitivity of the results to the time integration algorithm that we experienced corroborates this point. Further investigation in this direction is needed and left for future work.

\section{A CRITICAL LOOK INTO RAYLEIGH DAMPING FORCES}

For accurate prediction of the inelastic response of structures in seismic loading, it would be ideal to have a reliable predictive model of the discrepancy forces. That is a model that would be capable of accurately accounting for the phenomena not explicitly represented in the hysteretic forces. Although there presently is a consensus in the earthquake engineering community that using Rayleigh damping forces is far from reaching this ultimate goal [3, 10], common implementation of inelastic time history seismic analyses adds Rayleigh damping forces to the hysteretic structural forces. Whether one can expect the damping forces to be a good model for the discrepancy forces or not is something that can now be discussed based on the rationale provided by the knowledge of the discrepancy forces associated to model $\mathcal{H}_{1}$ or $\mathcal{H}_{2}$ acquired in the previous sections.

7.1. Forces in seismic simulations with Rayleigh damping. Inelastic structural models $\mathcal{H}_{1}$ and $\mathcal{H}_{2}$ are now used along with a Rayleigh damping model to run seismic simulations of the frame. Because the frame is dominated by its first 

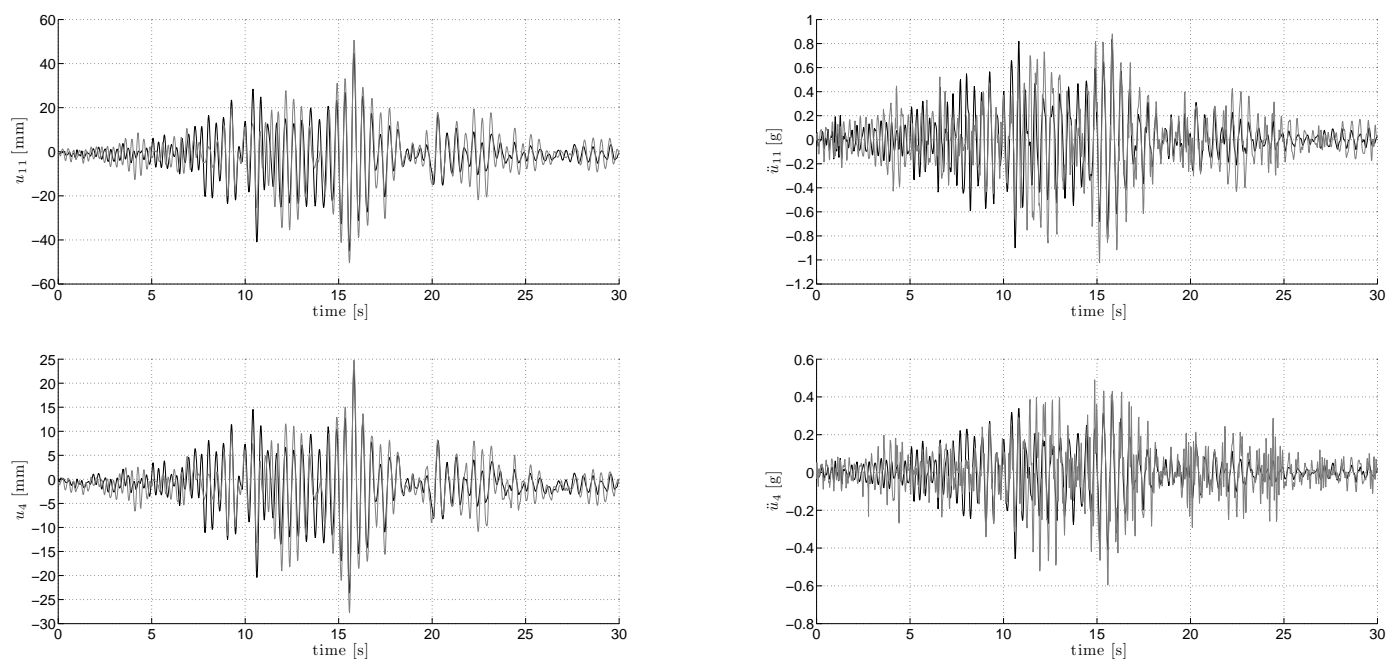

FiguRE 15. [top] $2^{\text {nd }}$-level relative displacement [left] and relative acceleration [right] time histories; [bottom] $1^{\text {st }}$-level relative displacement [left] and relative acceleration [right] time histories. Experimental data (plain line / black) and numerical simulation with model $\mathcal{G}_{2}$ (dashed line with markers).

mode and the structure is stiff $\left(T_{1}^{\mathcal{H}_{1}}=T_{1}^{\mathcal{H}_{2}}=0.29 \mathrm{~s}\right)$, we adopt hereafter the mass-proportional version of Rayleigh damping. Accordingly, model $\mathcal{D}$ is build as:

$$
\mathbf{F}^{d i s}(t)=\alpha \mathbf{M} \dot{\mathbf{u}}(t)
$$

where $\dot{\mathbf{u}}$ is the velocities vector.

Coefficient $\alpha$ is computed with the help of the following relation that links the damping ratio $\xi$ to the circular frequency $\omega$ pertaining to mode $m$ at time $t$ during the time history seismic analysis (see e.g. [13]):

$$
\xi_{m}(t)=\frac{\alpha}{2 \omega_{m}(t)} .
$$

Accordingly, damping ratio is expected to increase while structure damages and its fundamental frequency decreases during strong seismic motion. Mass-proportional Rayleigh damping designed with a target damping ratio for mode $1 \hat{\xi}_{1}=3 \%$, that is $\alpha=1.1702 \mathrm{~s}^{-1}$, is found to provide relatively satisfactory results in terms of peak displacement and acceleration, for both models $\mathcal{H}_{1}$ and $\mathcal{H}_{2}$, as shown in figures 16 and 17.

Here are some further details on the notion of targeted damping ratio $\hat{\xi}_{1}$. Before seismic loading is imposed and after static loading has been applied, both structural models $\mathcal{H}_{1}$ and $\mathcal{H}_{2}$ have fundamental circular frequency $\omega_{1}(0)=21.67 \mathrm{rad} . \mathrm{s}^{-1}$, 

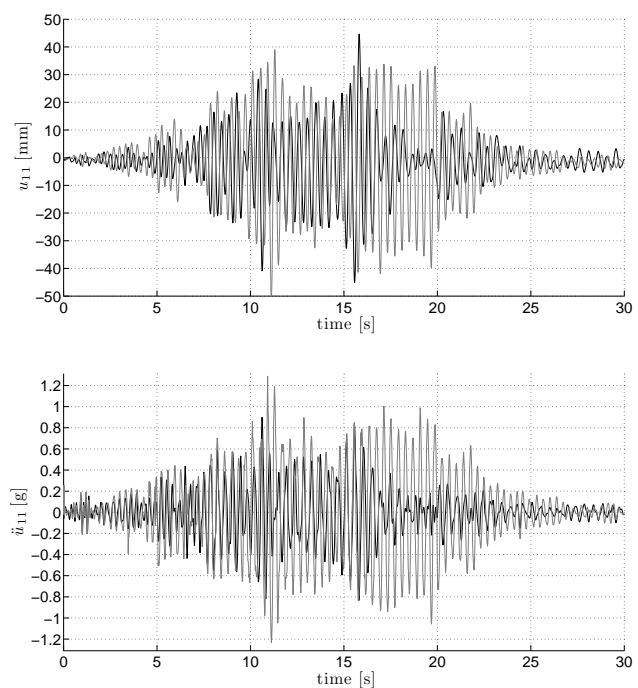

FIGURE 16. Experimental (black) and simulated (grey) $2^{\text {nd }}$-level relative displacement [top] and relative acceleration [bottom] time histories. Simulations are run with model $\mathcal{H}_{1}$ along with massproportional Rayleigh damping $\left(\hat{\xi}_{1}=3 \%\right)$.
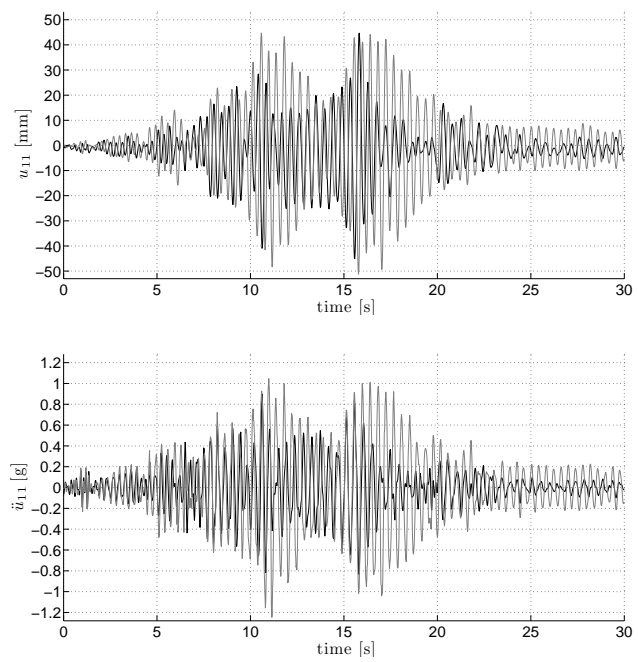

FiguRE 17. Experimental (black) and simulated (grey) $2^{\text {nd }}$-level relative displacement [top] and relative acceleration [bottom] time histories. Simulations are run with model $\mathcal{H}_{2}$ along with massproportional Rayleigh damping $\left(\hat{\xi}_{1}=3 \%\right)$. 

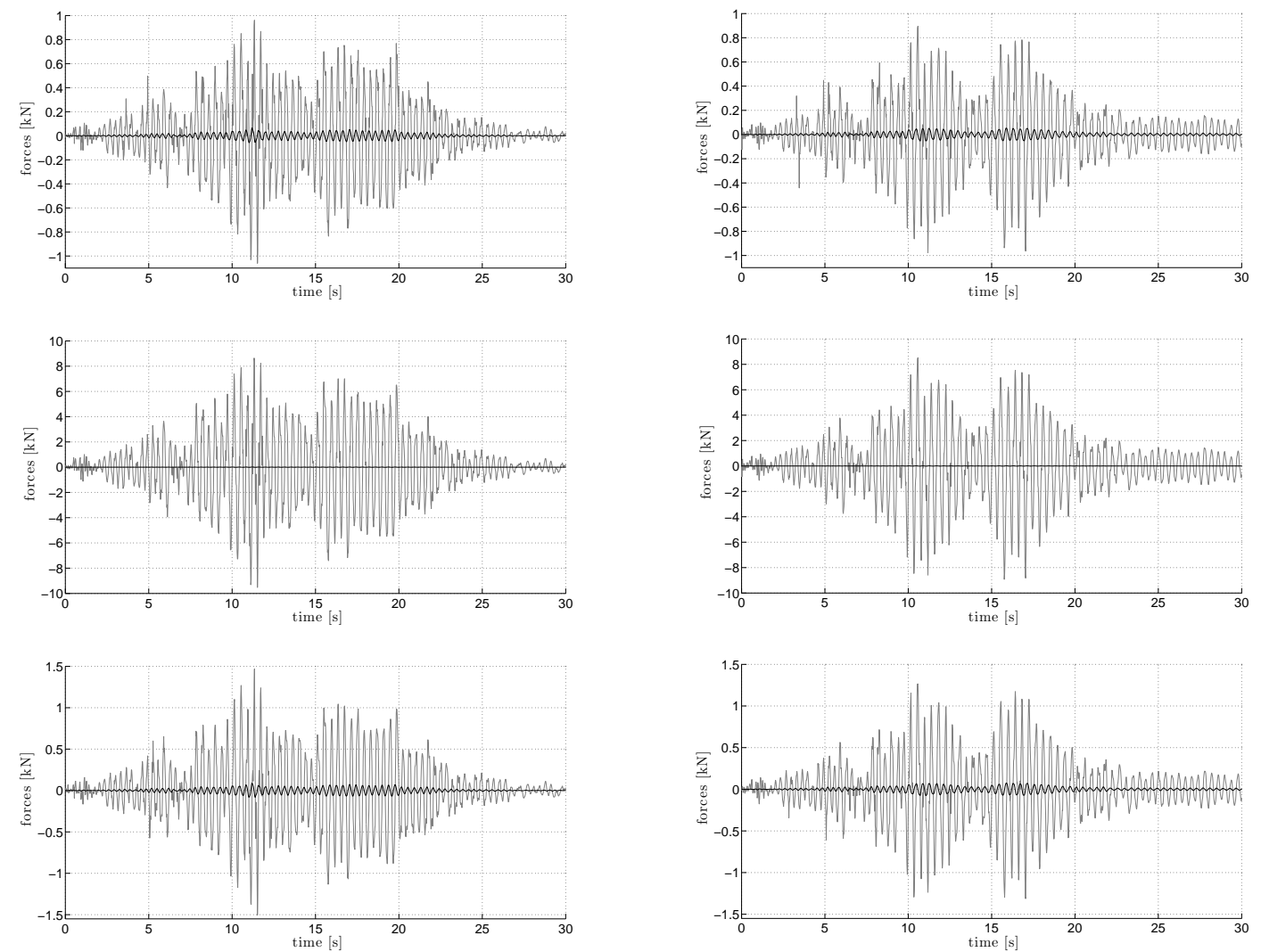

FiguRE 18. Damping (black) and hysteretic (grey) forces time histories simulated using model $\mathcal{H}_{1}$ [left] and $\mathcal{H}_{2}$ [right] along with mass-proportional Rayleigh damping $\left(\hat{\xi}_{1}=3 \%\right)$ at nodes 4 [top], 5 [center], and 7 [bottom]. At node 5, horizontal velocity being almost identical all along the $1^{\text {st }}$-level beam, damping forces are almost null.

that is, according to equation (16) with $\alpha=1.1702 \mathrm{~s}^{-1}, \xi_{1}(0)=2.7 \%$. After seismic motion, for structure $\mathcal{H}_{1}: \omega_{1}^{\mathcal{H}_{1}}(T)=17.95 \mathrm{rad} . \mathrm{s}^{-1}$ and $\xi_{1}^{\mathcal{H}_{1}}(T)=3.3 \%$, and for structural model $\mathcal{H}_{2}: \omega_{1}^{\mathcal{H}_{2}}(T)=16.11 \mathrm{rad} . \mathrm{s}^{-1}$, that is $\xi_{1}^{\mathcal{H}_{2}}(T)=3.7 \%$.

Besides, Rayleigh damping is implemented so that no contribution to the massproportional damping term comes from the elements that support the additional masses (elements 8, 11, 14 and 17) to avoid any artificial effects due to the concentration of mass in these regions of the frame.

The hysteresis and damping forces time histories observed during these simulations are shown in figure 18 for model $\mathcal{H}_{1}$ [left] and model $\mathcal{H}_{2}$ [right]. The amplitudes are here much smaller than those observed for the discrepancy forces in figure 11. Mass-proportional Rayleigh damping forces remain small comparing with the hysteretic forces for both models $\mathcal{H}_{1}$ and $\mathcal{H}_{2}$, which is often considered 
as a requirement for a good modeling of damping in other works $[10,3]$. Using the same argument as previously, a perfect structural model $\mathcal{H}$ would not require any additional forces to satisfy the equilibrium equation and, therefore, the presence of mass-proportional Rayleigh damping forces illustrates the fact that some phenomena are missed by the structural model. However, these latter forces are much smaller than the discrepancy forces observed in figure 11: this shows that these mass-proportional Rayleigh damping forces do not provide as sharp an insight into the structural model $\mathcal{H}$ deficiencies.

Note that the same analysis has also been performed with both mass and tangent stiffness-proportional terms for the Rayleigh damping model $\left(\mathbf{F}^{\text {dis }}(t)=\right.$ $(\alpha \mathbf{M}+\beta \mathbf{K}(t)) \dot{\mathbf{u}}(t)$, where $\mathbf{K}(t)$ is the structural tangent stiffness matrix) and with a targeted damping ratio $\hat{\xi}=3 \%$ for the first two modes of the frame. With this procedure, damping matrix $\mathbf{C}(t)=\alpha \mathbf{M}+\beta \mathbf{K}(t)$ is modified at each time step of the analysis according to the modification of the stiffness matrix. Coefficients $\alpha$ and $\beta$ are computed once for all at the beginning of the the analysis. Using this other damping model yields the same conclusions as previously.
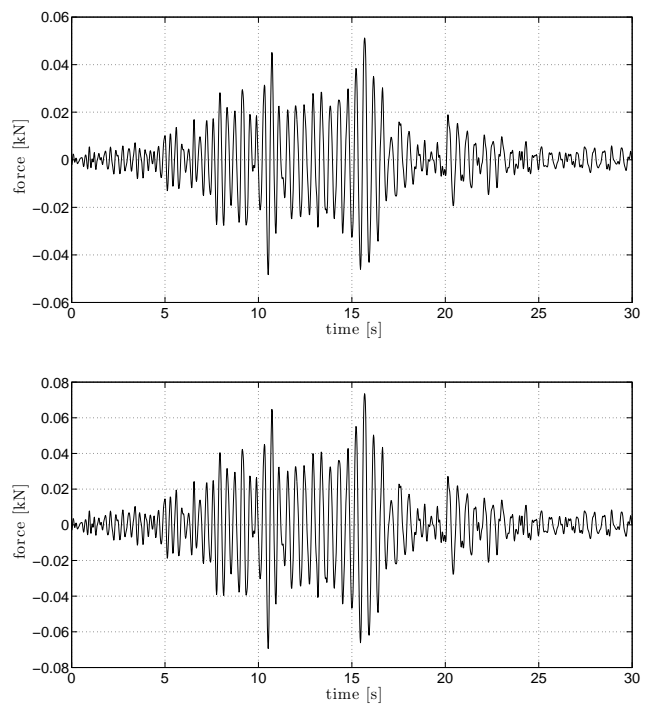

FIgURE 19. Rayleigh damping-like discrepancy forces consistent with the experimental data. Forces are simulated with massproportional Rayleigh damping $\left(\hat{\xi}_{1}=3 \%\right)$ at nodes 4 [top] and 7 [bottom]. Mass-proportional damping forces are independent of the structural model because stiffness matrix is not considered. Forces at node 5 are null because same horizontal velocity is assumed all over $1^{\text {st }}$-level beam. 


\subsection{Rayleigh damping forces consistent with the experimental data.}

Keeping the same damping model as above, we now compute Rayleigh damping forces that are consistent with the experimental displacement time history. The velocities are approximated as the first time derivative of the experimental displacement time history: $\dot{\mathbf{y}}_{n}^{e} \approx\left(\mathbf{y}_{n+1}^{e}-\mathbf{y}_{n}^{e}\right) / \Delta t$, and resulting mass-proportional Rayleigh damping forces are shown in figure 19. Recalling that the appropriate discrepancy forces can reach up to $100 \mathrm{kN}$ (see figure 11), this illustrates that mass-proportional Rayleigh damping forces are inconsistent with the hysteretic forces induced by both inelastic structural models $\mathcal{H}_{1}$ and $\mathcal{H}_{2}$. In other terms, the equations $\tilde{\mathbf{R}}\left(\mathbf{y}^{e}, \dot{\mathbf{y}}^{e}, \ddot{\mathbf{y}}^{e}\right)=\mathbf{0}$ has no solution when the discrepancy forces are modeled as mass-proportioanl Rayleigh damping forces.

\section{Conclusions}

In this paper, the general concept of discrepancy forces has been introduced in the framework of computational dynamics. Given a structural model and experimental data, discrepancy forces are defined as the forces needed to satisfy the equilibrium equation when the experimental data are imposed to the structural model.

Rayleigh damping model is commonly added to the structural model in seismic time history analyses of structures to account for internal energy absorption mechanisms not otherwise explicitly represented by the structural model. It has been shown in the literature that Rayleigh damping is difficult to control throughout inelastic analyses and that, besides, the conclusions of seismic performance studies can be significantly affected by uncertain Rayleigh damping forces. Better knowledge of how additional damping forces should be computed thus is highly desirable. To that purpose, we propose in this paper to consider Rayleigh damping forces as a model of discrepancy forces rather than as intrinsic component of the structural response (which is done by systematically adding Rayleigh damping forces in the equilibrium equation).

The purpose of this proposed shift of perspective is to provide a critical look into Rayleigh damping forces. Given experimental data, it is possible to calculate the discrepancy forces, which provides the rationale for a critical analysis of Rayleigh damping forces as a candidate for modeling these discrepancy forces. It would indeed be ideal to have predictive models of the discrepancy forces for a given structural model and we thus investigated whether Rayleigh damping forces could meet this ultimate goal or not.

In this work, it has been observed that, for the reinforced concrete momentresisting frame structure considered:

- The Rayleigh damping model used is not an adequate model for the discrepancy forces;

- While discrepancy forces provide insight into the capability of the structural model for representing the actual structural behavior, the modeled Rayleigh 
damping forces only shed light on the structural model to a more limited extent;

- The forces developed by the structural model can be strongly different according to whether the computed discrepancy forces or Rayleigh damping forces are introduced in the simulations.

This work also points out that, in analyses where structural forces are combined with damping forces, the validity of a structural model can only be studied with respect to the damping model, and vice versa. Therefore, a better knowledge of the damping forces is relative to the structural model used.

As a final remark, this work illustrates how experimental data can be effectively used to identify the forces that are usually expected to be represented by Rayleigh damping in seismic structural analyses. In this sense, it could pave the way for better knowledge of damping forces.

\section{ACKNOWLEDGEMENT}

This research was supported by a Marie Curie International Outgoing Fellowship within the 7th European Community Framework Programme (proposal No. 275928). This support is gratefully acknowledged.

\section{REFERENCES}

[1] Ozan Cem Celik and Bruce R. Ellingwood. Modeling beam-column joints in fragility assessment of gravity load designed reinforced concrete frames. Journal of Earthquake Engineering, 12:357-381, 2008.

[2] Ozan Cem Celik and Bruce R. Ellingwood. Seismic fragilities for non-ductile reinforced concrete frames - Role of aleatoric and epistemic uncertainties. Structural Safety, 32:1-12, 2010 .

[3] Finley A Charney. Unintended consequences of modeling damping in structures. ASCE Journal of Structural Engineering, 134(4):581-592, 2008.

[4] Luc Davenne, Frédéric Ragueneau, Jacky Mazars, and Adnan Ibrahimbegovic. Efficient approaches to finite element analysis in earthquake engineering. Computers and Sructures, 81:1223-1239, 2003.

[5] Matjaz Dolsek. Incremental dynamic analysis with consideration of modeling uncertainties. Earthquake Engineering and Structural Dynamics, 38:805-825, 2009.

[6] Bruce R. Ellingwood and Kursat Kinali. Quantifying and communicating uncertainty in seismic risk assessment. Structural Safety, 31:179-187, 2009.

[7] André Filiatrault, Éric Lachapelle, and Patrick Lamontagne. Seismic performance of ductile and nominally ductile reinforced concrete moment resisting frames. I. Experimental study. Canadian Journal of Civil Engineering, 25:331-341, 1998.

[8] André Filiatrault, Éric Lachapelle, and Patrick Lamontagne. Seismic performance of ductile and nominally ductile reinforced concrete moment resisting frames. II. Analytical study. Canadian Journal of Civil Engineering, 25:342-352, 1998.

[9] Roger Ghanem and Masanobu Shinozuka. Structural-system identification. I: Theory. Journal of Engineering Mechanics, 121(2):255-264, 1995.

[10] J F Hall. Problems encountered from the use (or misuse) of Rayleigh damping. Earthquake Engineering and Structural Dynamics, 35:525-545, 2006. 
[11] Hans M Hilber, Thomas J R Hughes, and Robert L Taylor. Improved numerical dissipation for time integration algorithms in structural dynamics. Earthquake Engineering and Structural Dynamics, 5:283-292, 1977.

[12] Pierre Jehel, Luc Davenne, Adnan Ibrahimbegovic, and Pierre Léger. Towards robust viscoelastic-plastic-damage material model with different hardenings / softenings capable of representing salient phenomena in seismic loading applications. Computers and Concrete, $7(4): 365-386,2010$.

[13] Pierre Jehel, Pierre Léger, and Adnan Ibrahimbegovic. Initial versus tangent stiffness-based Rayleigh damping in inelastic time history seismic analyses. Earthquake Engineering and Structural Dynamics, 43:467-484, 2014.

[14] Patrick Lamontagne. Comportement sismique d'une ossature en béton armé ( $\mathrm{R}=4)$. Master's thesis, École Polytechnique de Montréal, Montréal, QC, Canada, January 1997.

[15] Tae-Hyung Lee and Khalid M Mosalam. Seismic demand sensitivity of reinforced concrete shear-wall building using FOSM method. Earthquake Engineering and Structural Dynamics, 34:1719-1736, 2005.

[16] Abbie B Liel, Curt B Haselton, Gregory G Deierlein, and Jack W Baker. Incorporating modeling uncertainties in the assessment of seismic collapse risk of buildings. Structural Safety, 31:197-211, 2009.

[17] Nilanjan Mitra and Laura N Lowes. Evaluation, calibration, and verification of a reinforced concrete beam-column joint model. Journal of Structural Engineering, 133(1):105-120, January 2007.

[18] Keith A Porter, James L Beck, and Rustem V Shaikhutdinov. Sensitivity of building loss estimates to major uncertain variables. Earthquake Spectra, 18(4):719-743, 2002.

[19] Bojana V Rosić, Anna Kučerova, Jan Sýkora, Oliver Pajonk, Alexander Litvinenko, and Hermann G. Matthies. Parameter identification in a probabilistic setting. Engineering Structures, 50:179-196, 2013.

[20] Masanobu Shinozuka and Roger Ghanem. Structural-system identification. II: Experimental verification. Journal of Engineering Mechanics, 121(2):265-273, 1995.

[21] Seymour M Spence and Ahsan Kareem. Tall buildings and damping: A concept-based datadriven model. Journal of Structural Engineering, 140(5):04014005, 2014.

[22] R.L. Taylor. FEAP: A Finite Element Analysis Program, User manual and Programmer manual. University of California, Berkeley, California, 2005. Version 7.4.

[23] E Tubaldi, Michele Barbato, and A Dall'Asta. nfluence of model parameter uncertainty on seismic transverse response and vulnerability of steel-concrete composite bridges with dual load path. Journal of Structural Engineering, 128(3):363-374, March 2012.

[24] Dimitrios Vamvatsikos and Michalis Fragiadakis. Incremental dynamic analysis for estimating seismic performance sensitivity and uncertainty. Earthquake Engineering and Structural Dynamics, 39:141-163, 2010. 